\title{
MOTORWAY NETWORK SIMULATION USING BLUETOOTH DATA
}

\author{
Ioannis Karakikes ${ }^{1}$, Matthias Spangler $^{2}$, Martin Margreiter ${ }^{3}$ \\ ${ }^{1}$ University of Thessaly \\ Pedion Areos, 38334, Volos, Greece \\ +302421074133, iokaraki@civ.uth.gr \\ ${ }^{2}$ Technical University of Munich \\ Arcistrasse 21, Munich, Germany \\ +498928922333, matthias.spangler@tum.de \\ ${ }^{3}$ Technical University of Munich \\ Arcistrasse 21, Munich, Germany \\ +498928928586, martin.margreiter@tum.de
}

This paper describes a systematic calibration process of a Vissim model, based on data derived from BT detectors. It also provides instructions how to calibrate and validate a highway network model based upon a case study and establishes an example for practitioners that are interested in designing highway networks with micro simulation tools. Within this case study, a $94,5 \%$ proper calibration to all segments was achieved First, an overview of the systematic calibration approach that will be followed is presented. A description of the given datasets follows. Finally, model's systematic calibration and validation based on BT data from segments under free flow conditions is thoroughly explained. The delivered calibrated Vissim model acts as a test bed, which in combination with other analysis tools can be used for potential future exploitation regarding transportation related purposes.

Keywords: simulation, highway network, Bluetooth data, Vissim, calibration

\section{Introduction}

Over the last decades, micro analytic simulation has turned out to be a valuable tool for planning, designing and evaluating transportation systems. Micro simulation provides a fast, safe, economic and reliable way of examining whether an 'intervention' on a current system could output the desired results or not. It has also been proved a valuable tool for testing multiple different traffic scenarios, visualization and evaluation of future conditions of a system. Micro simulation mainly focuses on the interaction between a car and the road, but with the necessary input datasets along with the respective experience of the practitioner, can be also used to simulate wider systems that normally would be examined meso- or macroscopically. On the market there are many tools which can be used for micro simulation. The most prevalent are Vissim from PTV, AIMSUN from TSS- Transport, CORSIM by US Federal Highway Administration and PARAMICS from Quadstone Limited. All models that will be designed with the aforementioned micro simulators need calibration, in order to produce credible and reliable output. Calibration is a prerequisite to replicate accurately the real traffic situations of a system.

Aim of this paper is to simulate a Bavarian highway network, formed by A70, A7, A6, A73, A9 and A3 highways based on Bluetooth (BT) data through a systematic calibration process. BT data in the sense of travel time measurements, can produce reliable output for a model. Along with the calibration process, a validation process to verify the credibility of the model under fresh field data is also described.

\section{Traffic Model's Calibration Process}

Many systematic and comprehensive calibration processes have been developed in order to standardize the calibration process of traffic models. The aim of these systematic approaches was to reduce the computational effort of the calibration process as well as to maximize the reliability of the exported results. However, these systematic procedures cannot produce reliable results in all situations. The main reason for this is the great variability of the traffic models and the different needs in every case that the practitioner has to cover. 
Except for the characteristics of the simulated model, another difficulty of developing a general systematic calibration process lies in the inability to set a rule to come up with the exact values of the parameters that must be adjusted in order to model precisely the driving behavior. Optimization algorithms are applied to reveal the optimum parameter set that will minimize the discrepancy between simulation results and field data. However, the number of the parameters that will be taken into consideration from the optimization algorithm, the range of parameters' values and the type of the parameters that influence the driving behavior still remain a nonstandard matter that cannot be restrained into generic systematic procedures.

This paper's objective is to present and explain a manual systematic approach for motorway networks' calibration, based on automatic parameters' adjustment from similar traffic models and literature. However, the following approach can be applied to various traffic model cases.

\subsection{Systematic Calibration Overview}

A micro simulation traffic model requires a variety of information as inputs such as information related to traffic volumes, desired speeds, driving behaviors, accelerations or even the duration of a potential temporary lack of attention of a driver. A model's level of fidelity is inextricably connected with all those data. The higher the number of information a practitioner disposes, the higher the level of the model's accuracy will be. In many cases however, the collection of all the necessary piece of information is rather difficult, either because the cost of the acquisition is becoming extremely high and will never be compensated, or just because several aspects of the human driving behavior cannot be easily recorded or quantified. Hence, the practitioner is called to fine-tune the model by adjusting certain parameters until the exported data fall within an acceptable divergence from the real field data.

An uncalibrated model cannot replicate with accuracy different traffic conditions. Calibration ensures that the developed model reproduces all the individual characteristics of a traffic situation, according to the targets that initially have been set. However, each micro simulation software package cannot contain as parameters, all the aspects of driving behavior. Hence, different parameters can be found in different traffic simulators. The analyst after several simulation runs, is responsible to come up with a certain set of parameters in order to achieve the desired proximity. The adjustment set of parameters process can either aim to a more general model calibration or to a more local one (link based, node based). Dowling et al. (2004) split the calibration strategy into three steps: the capacity calibration of the model, the route choice calibration and the overall system calibration performance. Under a different approach So et al. (2015) used volume checks, speed checks and travel time checks as a calibration strategy.

There are many different manual or automatic approaches in the literature that can be followed to describe a calibration process. Manual approaches are based on multiple iterations that meant to close the gap between the simulation output and the real data (trial and error process), while automatic approaches are based on minimization of fitness functions' values (based on algorithms). Three of these approaches by Park et al. (2006), Chu et al. (2004) and Hourdakis et al. (2003) are described below. The first (Park et al., 2006) distinguish an automatic calibration into the following seven steps:

1. 'Building' the model and loading all the necessary elements on it, according to every simulators user's manual.

2. Checking the feasibility of the model without changing the default parameters. Except for rare occasions, a model always needs further adjustment to comply with the field conditions. If that's the case we proceed to the third step.

3. Sensitivity analysis for every parameter and determination of an acceptable parameter range. The desired range and the feasibility of a parameter can be determined by the information coming out of the field data using all the methods that the practitioner has in his disposal.

4. Final optimal selection of parameters, from their predefined ranges through the deployment of a Genetic algorithm.

5. Checking the performance of the adjusted parameters by running multiple times the simulation. The result should be corresponding to the observed/counted/given field data in all simulation runs and by all different random seeds.

6. Making an animation and then reassuring visually that there are no traffic behavior abnormalities.

7. Comparison of the exported data with a whole new field data set (even on a different day, as long as it fulfills the 'typical day' preconditions, see subchapter 2.3). This check called 'Validation'. Given that the previous steps were realized successfully, exported data should comply with the targets that have been initially set. 
Chu et al. (2004) describe a manual systematic calibration process of four steps:

1. Calibration of driving behaviors

2. Calibration of route choice model

3. Estimation of the OD Matrix

4. Final fine tuning of the model

Hourdakis et al. (2003) categorize a systematic manual calibration in three steps:

1. Calibration based on traffic volumes

2. Calibration based on travel speeds

3. Calibration based on the objectives of a model

The calibration process along with the preparation, initial modeling, simulation experiment and documentation phase is presented in the following flowchart, according to the Research Society for Roads and Transportation in Germany (FGSV, 2006), see Figure 1. Many different adapted versions of this flowchart can be found, developed by several institutions.

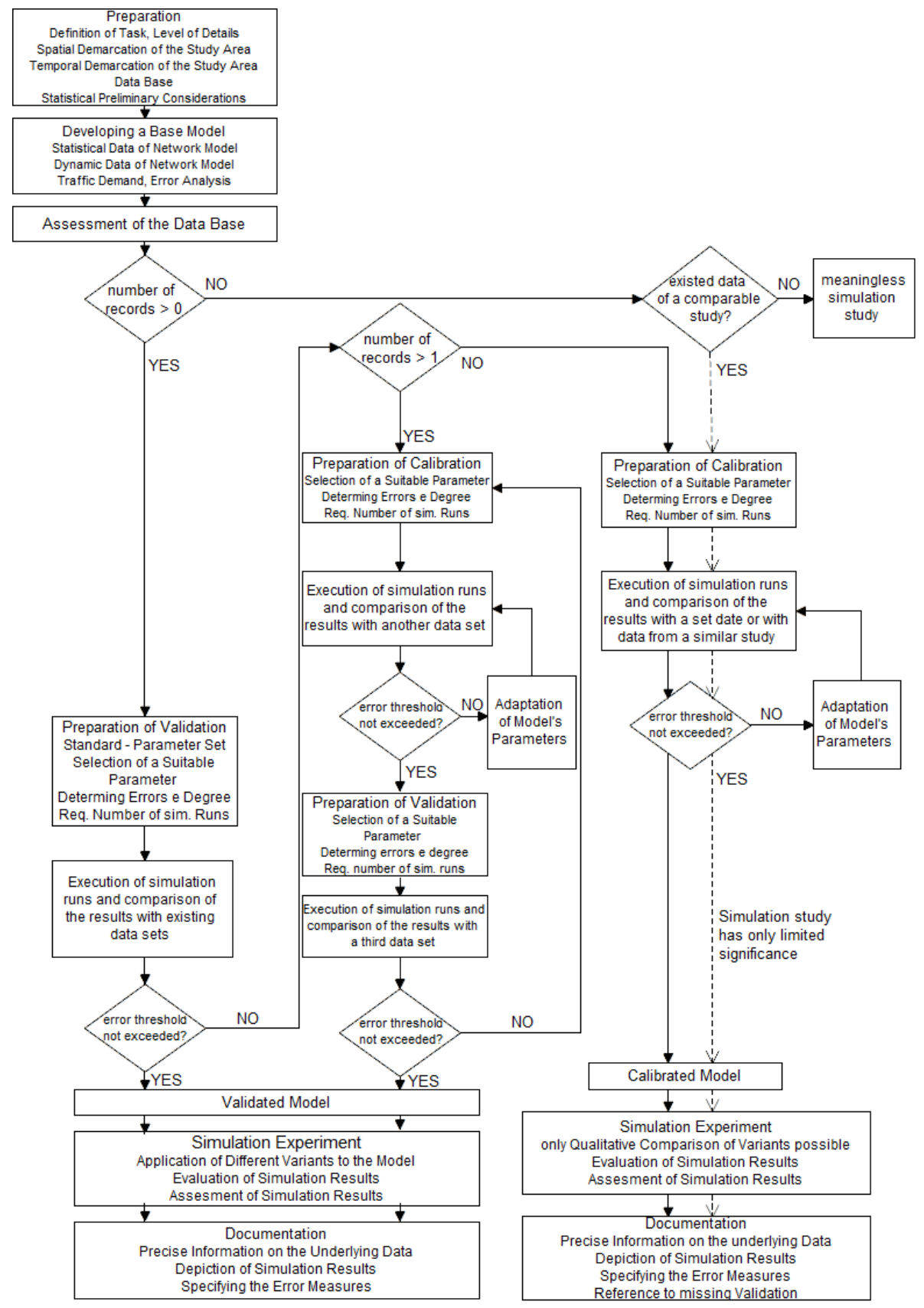

Figure 1. Flowchart of a simulation study (translated in English) (FGSV Verlag, 2006) 


\subsection{Calibration based on Bluetooth Data}

Travel times for motorway models are the most dominant and widely-used way for calibration, due to their extraction easiness from Bluetooth (BT) detectors, Automatic Number Plate Recognition (ANPR), floating vehicle data etc. Travel time data also enclose a great variety of information about the traffic situation especially under low or normal flow conditions. In this model travel times are derived from BT detectors.

\subsection{Calibration Targets and Typical Day}

Calibration is a repetitive procedure until the desired correspondence between the field data and model's results will be eventually achieved. Nevertheless, perfect matching is nearly impossible, since the number of iterations in order to eliminate a re-occurring error cannot be infinite. Analyst's trials should be stopped when certain deviations are reached, according to the initial calibration thresholds that have been set. Those thresholds can be different and mostly depending on the desired level of fidelity that a project commands, based on the resources that are provided (Dowling et al., 2004). A prevalent example of calibration criteria is presented by the Wisconsin Department of Transportation (2002).

Table 1. Calibration Criteria (Wisconsin Department of Transportation, 2002)

\begin{tabular}{|l|l|}
\hline Criteria and Measures & Calibration Acceptance Targets \\
\hline Hourly Flows, Model Versus Observed & $>85 \%$ of cases \\
\hline Individual link flows & $>85 \%$ of cases \\
\hline Within $15 \%$, for $700 \mathrm{vph}<$ flow $<2700 \mathrm{vph}$ & $>85 \%$ of cases \\
\hline Within $100 \mathrm{vph}$, for flow $<700 \mathrm{vph}$ & Within $5 \%$ of sum of all links counts \\
\hline Within 400 vph, for flow $>2700 \mathrm{vph}$ & $>85 \%$ of cases \\
\hline Sum of all link flows & GEH $<4$ for sum of all link counts \\
\hline GEH statistic $<5$ for individual link flows & $>85 \%$ of cases \\
\hline GEH statistic for sum of all link flows & to analyst's satisfaction \\
\hline \multicolumn{2}{|l}{} \\
\hline Travel Times, Model Versus Observed & to analyst's satisfaction \\
\hline Journey times network within $15 \%$ (or 1 min, if higher) & \\
\hline \multicolumn{2}{|l|}{} \\
\hline Visual Audits & \\
\hline Individual link speeds: & \\
\hline Visually acceptable speed-flow relationship & Bottlenecks: \\
\hline Visually acceptable queuing & \\
\hline
\end{tabular}

The GEH statistic has been named after the initial letters of Geoffrey E. Havers and is an empirical formula widely used for traffic analysis purposes. The advantage of this formula is that it sets different tolerance percentages according to various traffic volumes (Wisconsin Department of Transportation, 2002). More specifically, a logical fixed $10 \%$ deviation tolerance on the mainline of a highway servicing $5000 \mathrm{veh} / \mathrm{h}$ would range from $4500 \mathrm{veh} / \mathrm{h}$ to $5500 \mathrm{veh} / \mathrm{h}$, while on a $50 \mathrm{veh} / \mathrm{h} \mathrm{ramp}$ would range from $45 \mathrm{veh} / \mathrm{h}$ to $55 \mathrm{veh} / \mathrm{h}$ which is rather stringent. GEH statistic acceptance threshold $(\mathrm{GEH}=5)$ would produce ranges of 4665-5355 veh/h and 21-93 veh/h respectively. GEH statistic's formula is similar to chi-squared test and can be computed as follows,

$G E H=\sqrt{\frac{(V-E)}{\frac{(E+V)}{2}}}$,

where:

$\mathrm{V}$ - counted volumes,

E - model's estimated volumes. 
All data necessary for the simulation are collected on the day of our interest in accordance with the scope of the modeling. Usually, this day is a weekday that includes the peak hours of a network (rare examples could be areas around, for example a zoo or a football field where the modeling interest shifts on weekends). However, second field data sets that can be used to calibrate and validate our network, are not always possible to be extracted the very same simulation day. In those cases, the new field data sets are derived from different days which are assumed to represent adequately the traffic behavior and characteristics of the simulation day. Nevertheless, due to traffic's variation and randomness the collected data may not represent a typical day. According to Wisconsin Department of Transportation (2002) the same GEH statistic formula adjusted, can guarantee at a satisfactory level the 'typical' character of the candidate day. The selection of a typical day can be implemented based on the comparison of the peak hour traffic volumes of a candidate day at any inductive loop with the average peak hour's volumes of all candidate days:

$G E H=\sqrt{\frac{(\text { Vi-Vaver })}{\frac{(\text { Vi }+ \text { Vaver })}{2}}}$,

where:

$\mathrm{V}_{\mathrm{i}}$ - volumes of candidate day,

$\mathrm{V}_{\text {aver }}$ - average volumes of all the candidate days.

If in the $85 \%$ of the selected detectors the GEH values are less than 5 , then the candidate day can be selected as a typical day.

\section{Model's Calibration and Validation}

\subsection{Vissim's Output}

Twelve vehicle travel time measurement points were placed precisely at the respective positions of the BT detectors on the simulated network. Those twelve points on the eight segments are named after the number of the BT detectors which define their start and their end. For every simulation run, one output file was exported. In these (*.RSR) files, Vissim generates raw travel time data in chronological order for every completed travel time measurement event (see PTV VISSIM 7 user's manual for further details). The exported vehicle travel times referred to nine travel time measurement sections based on Bluetooth's travel time data.

\subsection{Calibration}

The calibration and validation processes for this model, are entirely based on travel times coming from BT detectors on 24.06.2014 and 25.06.2014 respectively (Margreiter et al., 2015). Aim of the calibration process is to minimize the discrepancy between travel times extracted from BT detectors and travel times which are exported from Vissim, while validation's process scope is to verify that the model produces reliable results. In order to achieve this, a certain combination set of parameters should be eventually selected based on logical reasoning and accurate traffic replication. The discrepancy between the travel times should be within $15 \%$ according to the calibration targets that have been initially set. The whole calibration process will rely on intervals where no incidents are involved, since calibration at segments under incident may vary from the desired free flow calibration approach of this paper.

Calibration based on travel times ought to be as detailed as possible. Therefore, average travel times aggregated every 30 minutes are exported from Vissim in order to measure the effectiveness of the calibration process. Cars' and heavy vehicles' average travel times will be compared with the respective real travel times from the BT detectors to see whether they deviate beyond the $15 \%$ threshold or not. The calibration process will be terminated once the travel times in all segments fall within the threshold. In order to normalize the results, five simulation runs with different random seeds will be performed (PTV Group, 2014) proposes a minimum requisite number of five simulation runs). The deviation of the two datasets is being computed as the average of the five absolute deviations of the respective time interval. 
DATASET Dev(i) $=\frac{\mid \operatorname{dev} \operatorname{run} 1 \text { (i) }|+| \operatorname{dev} \text { run } 2 \text { (i) }|+| \operatorname{dev} \text { run } 3 \text { (i) }|+| \operatorname{dev} \text { run } 4 \text { (i) }|+| \operatorname{dev} \text { run } 5 \text { (i) } \mid}{5}$

It has to be pointed out here, that a potential excess of the $15 \%$ threshold in travel times could be attributed not only to insufficient calibration but also to higher volumes on 23.06.2015 in comparison with the volumes on 24.06.2014. Those two days were considered typical owing to that both are Tuesdays, belong to the same month, are from two consecutive years in which no big structural interventions took place and finally, because the weather conditions were perfect in the $62,5 \%$ of the stations along the network. In addition, the independency of the eight segments guarantees, that potential higher volumes on one segment won't affect the performance on the adjacent. In case one produces significantly higher travel times deviations compared to other simulated segments, then incompatibility of the data (traffic volumes, speed travels, heavy vehicle percent and travel times) should be suspected and calibration process should be terminated without reaching the desired deviations in this particular segment.

\section{First Stage Calibration}

The first calibration stage concerns the reasonable adjustments of the input data (desired speeds) in order to minimize the discrepancy between the exported travel time measurements from Vissim and the travel time measurements from BT detectors on 24.06.2015, see Figure 2.

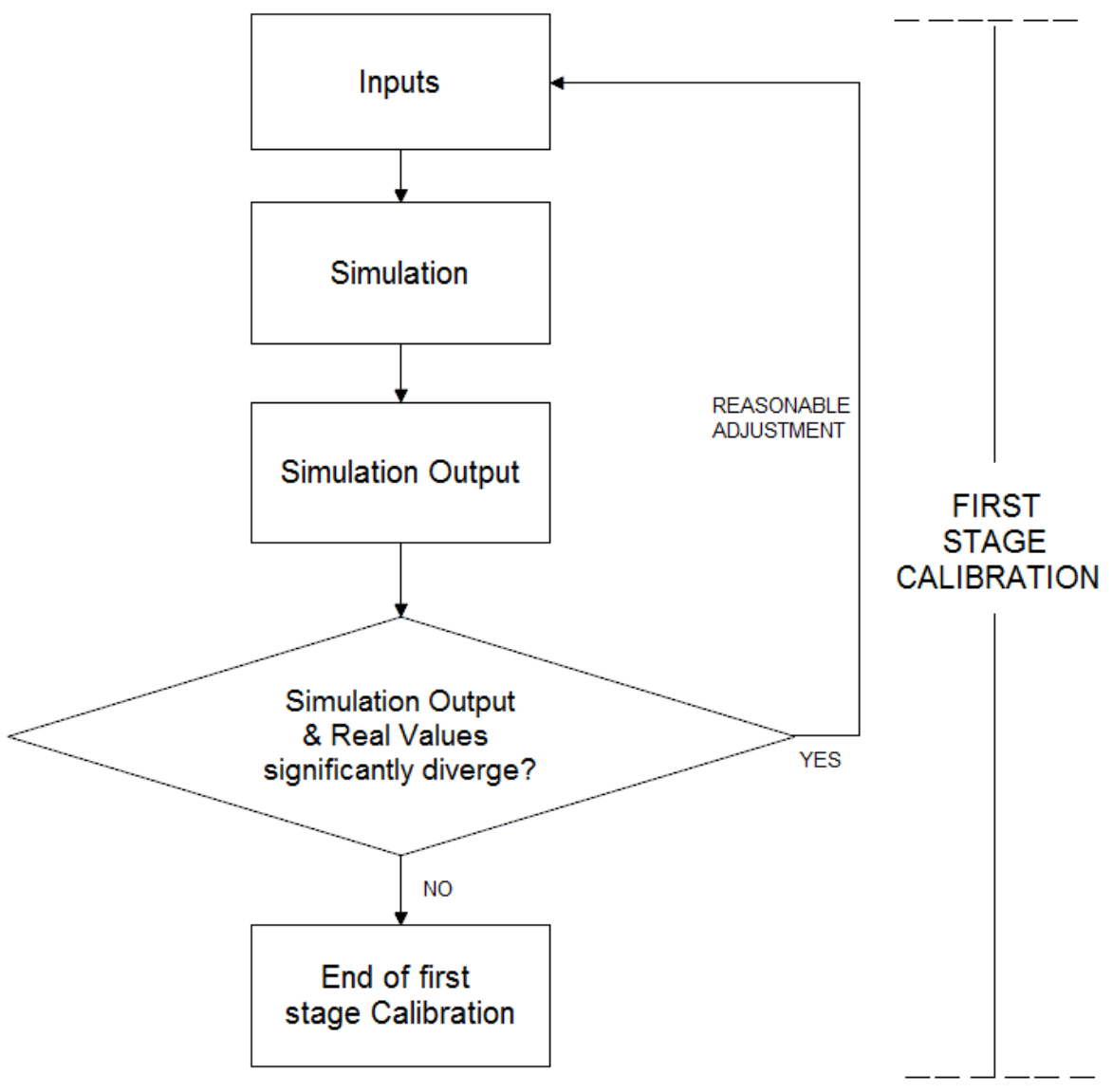

Figure 2. First stage model calibration - Inputs adjustment (Paz et al., 2015)

The criterion that was used to determine whether the simulation output of a section, during an interval, significantly diverge or not, is the sign of the respective travel time deviations in all five simulation runs. More specifically, if the respective deviations in all five simulation runs, present always the same sign (positive or negative), adjustments were made to the respective desired speed categories. Otherwise desired speed categories remained as they were. 


\section{Second Stage Calibration}

Aim of the second calibration stage is to find the optimum parameter set, which will be used to finetune the Vissim model, see Figure 3.

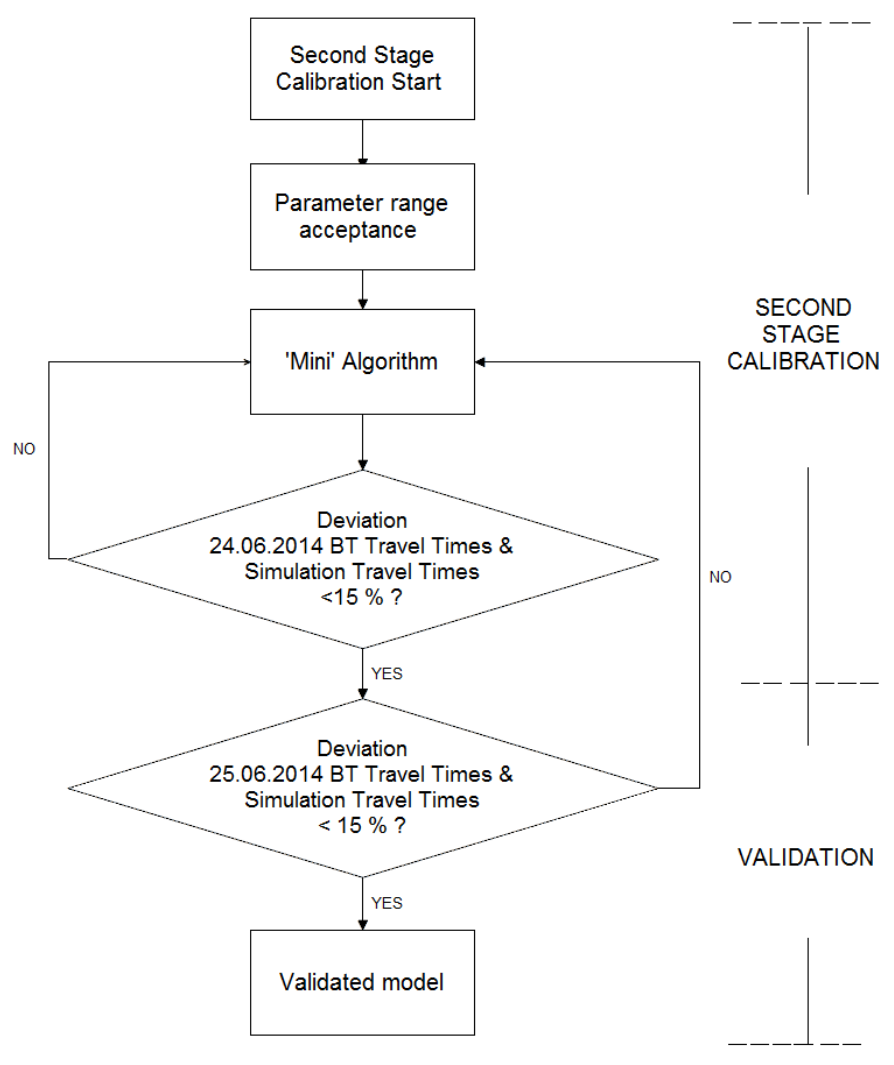

Figure 3. Second calibration stage - Parameters adjustment and Validation

Initially the parameters adjusted in order to replicate the simulated network's behaviour, were based on findings of a genetic algorithm (Xin, 2013) which was developed for a $4.820 \mathrm{~m}$ segment of the A9 Highway north of the city of Munich. Scope of this master thesis was to develop a genetic algorithm which would conclude to a set of parameters that provide reliable results for models simulating German highways. The two models have a lot of similarities as both models' main focus was to simulate the traffic on on/off ramps, without occurrence of traffic incidents. Xin (2013) found out that the evaluation criteria were sensitive mainly to five parameters: the 'Headway time' (CC1), the 'Following Variation' (CC2), the maximum deceleration of the trailing vehicle during a lane change, the safety distance parameters and the maximum deceleration of the trailing vehicle for a cooperative lane change.

After running five times the simulation, the majority $(94,4 \%)$ of the travel time deviations fall within the $15 \%$ threshold. However, the values for the car following behavior (CC1 and CC2) were considered unsuitable. Although, the combination of $0,5 \mathrm{sec}$ for $\mathrm{CC} 1$ and $1 \mathrm{~m}$ for $\mathrm{CC} 2$ do belong to the typical ranges that are given in the literature, such a short 'Headway Time' along with a tight 'Following Variation' are not representing the driving behavior on German highways under normal flow conditions.

Other papers by Gomes et al. (2004) and Rompis et al. (2014), referring also to highway model calibration, conclude to values for CC1 around 1.5 while concerning the CC2 parameter, Rompis et al. (2014) claim a value of 7,5. Although those highway networks are significantly larger than the one Xin (2013) examined and closer to the size of this network, they are more oriented towards calibration of high congested highway networks. Moreover, these parameters' values in combination with the previous lane change parameters were applied to this network, but the general performance of the simulation was characterized overcautious and unnecessarily conservative, especially to segments with light traffic volumes between 6:00 am and 8:00 am (12\% of the travel time sections deviate beyond the $15 \%)$.

Judging from the two previous approaches which were considered too 'aggressive' and too 'defensive' respectively, it was obvious that a value that would replicate accurately the driving behavior 
comprehensively should be found in between the values of $0,5-1,5 \mathrm{sec}$ for $\mathrm{CC} 1$ and $1-7,5 \mathrm{~m}$ for CC2. However, no further reference or logical justification could be provided to argue upon the selection of specific values between the upper ranges. This was determined through the development of a 'mini' algorithm for the model based on these two parameters. To come up with the exact parameters' values, possible combinations within the two boundaries were considered.

Two simulation runs with different random seeds were performed for every possible combination. Best fit for the calibration was combination with $\mathrm{CC} 1=0.9 \mathrm{sec} \& \mathrm{CC} 2=3 \mathrm{~m}$ which was run three additional times and gave the final average deviation results (still 5 out of 92 travel time deviations were exceeding the $15 \%$ threshold, see Table 2). A closer look on results indicated that sections between BT 68-86 at A6 segment during 8:00 am - 9:00 am and BT 91-90 at A3b segment during 7:30 am - 9:00 am are repetitively not complying with the set targets. Hence, we assume that the datasets that have been used to simulate the traffic conditions are incompatible to this segment and there is no need to calibrate further the model.

Table 2. Average absolute divergence percentages of travel times in $30 \mathrm{~min}$ intervals

\begin{tabular}{|c|c|c|c|c|c|c|c|c|c|c|c|c|c|c|}
\hline \multicolumn{15}{|c|}{ Average divergence percentage from Real Travel Times } \\
\hline \multirow{4}{*}{ 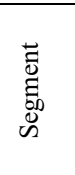 } & & & \multicolumn{12}{|c|}{ Percentage $\%$} \\
\hline & & & \multirow{2}{*}{\multicolumn{2}{|c|}{$\begin{array}{c}6: 00-6: 30 \\
5400-7200\end{array}$}} & \multicolumn{2}{|c|}{$6: 30-7: 00$} & \multicolumn{2}{|c|}{$7: 00-7: 30$} & \multicolumn{2}{|c|}{$7: 30-8: 00$} & \multicolumn{2}{|c|}{$8: 00-8: 30$} & \multicolumn{2}{|c|}{$8: 30-9: 00$} \\
\hline & & & & & \multicolumn{2}{|c|}{$7200-9000$} & \multicolumn{2}{|c|}{$9000-10800$} & \multicolumn{2}{|c|}{$10800-12600$} & \multicolumn{2}{|c|}{$12600-14400$} & \multicolumn{2}{|c|}{$14400-16200$} \\
\hline & At & To & Cars & HV & Cars & HV & Cars & $\mathrm{HV}$ & Cars & HV & Cars & $\mathrm{HV}$ & Cars & HV \\
\hline A70 & & ata) & & & & & & & o data & & & & & \\
\hline \multirow{3}{*}{ A7 } & & ata) & \multicolumn{12}{|c|}{ (no data) } \\
\hline & 93 & 94 & 1,5 & 6,0 & 1,6 & 7,6 & 1,9 & 2,3 & 2,9 & 5,8 & 0,8 & 6,9 & 5,4 & 2,5 \\
\hline & 94 & 85 & 3,4 & 3,9 & 4,0 & 3,9 & 5,7 & 3,6 & 1,0 & 2,8 & 4,7 & 5,0 & 8,1 & 5,3 \\
\hline \multirow{3}{*}{ A6 } & 83 & 82 & 4,0 & 5,6 & 8,1 & 7,4 & 6,4 & 1,2 & 10,4 & 2,7 & 13,6 & 1,6 & 14,2 & 0,8 \\
\hline & 82 & 68 & 5,4 & 3,7 & 11,3 & 1,5 & 5,0 & 2,8 & 10,7 & 2,4 & 11,6 & 5,2 & 14,2 & 3,2 \\
\hline & 68 & 86 & 12,5 & 4,3 & 6,2 & 1,7 & 8,1 & 2,7 & 9,8 & 3,9 & 15,7 & 6,3 & 15,5 & 5,5 \\
\hline A73 & 86 & 87 & \multicolumn{12}{|c|}{ (no data) } \\
\hline \multirow[t]{2}{*}{ A9 } & 87 & 47 & \multicolumn{12}{|c|}{ (no data) } \\
\hline & 47 & 81 & 1,3 & 1,1 & 2,0 & 1,1 & 7,3 & 2,2 & 8,0 & 1,3 & 3,1 & 1,9 & 0,7 & 0,9 \\
\hline A3 & 78 & 89 & \multicolumn{12}{|c|}{ (no data) } \\
\hline A73b & \multicolumn{2}{|c|}{ (no data) } & \multicolumn{12}{|c|}{ (no data) } \\
\hline \multirow{3}{*}{$\mathrm{A} 3 \mathrm{~b}$} & 92 & 91 & 2,7 & 3,0 & 4,0 & 4,9 & 2,7 & 3,3 & 6,9 & 4,4 & \multicolumn{4}{|c|}{ (no data) } \\
\hline & 91 & 90 & 6,4 & 1,4 & 8,8 & 3,2 & 5,9 & 1,3 & 16,7 & 1,0 & 23,3 & 1,7 & 28,4 & 3,3 \\
\hline & 90 & 89 & & & & & & & data & & & & & \\
\hline
\end{tabular}

\subsection{Validation}

Having all errors wiped out and simulation parameters determined, the calibrated travel times will be compared to a fresh travel time dataset on 25.06.2014. A properly calibrated model ought to produce travel times within the thresholds that have been set, otherwise it needs further calibration. Validation process is the second last step of a simulation study (before documentation, see flowchart in subchapter 2.1 and therefore, is the one that ultimately verifies that a model outputs reliable data which can be used for various purposes. However, before examining if the travel time deviations lie within the thresholds, the typicality of the new travel time dataset on 25.06.2014 should be checked.

Typicality of 25.06.2014 will be proved through the GEH criterion. Since the traffic volumes on this day are not available, the comparison of the two datasets will be based on the number of BT detections. Due to the non-linear behaviour of the GEH formula, the comparison of the number of the BT detections cannot be performed directly. According to Young (2012) in the United States an average $5 \%$ of the vehicles are carrying an active BT device. This percentage is indicative and coming from the observation that $400 \mathrm{BT}$ detections/h should almost be equivalent to $1000 \mathrm{veh} / \mathrm{h}$ which is an average volume at many sections from 6:00 am to 7:00 am. Assuming that the percentage of vehicles carrying an active BT device in the study area is $30 \%$, the GEH values in average at every segment for both cars and vehicles are (GEH values should be only computed in hourly volumes): 
Table 3. GEH values for 'typicality' determination between $24.06 .2014 \& 25.06 .2014$

\begin{tabular}{|l|l|l|l|}
\hline Segment no. & $\begin{array}{l}\text { GEH values for traffic } \\
\text { volumes between } \mathbf{2 4} \& \\
\mathbf{2 5 . 0 6 . 2 0 1 4 , ~ 6 : 0 0 - 7 : 0 0}\end{array}$ & $\begin{array}{l}\text { GEH values for traffic } \\
\text { volumes between 24 \& } \\
\mathbf{2 5 . 0 6 . 2 0 1 4 , ~ 7 : 0 0 - 8 : 0 0}\end{array}$ & $\begin{array}{l}\text { GEH values for traffic } \\
\text { volumes between 24 \& } \\
\mathbf{2 5 . 0 6 . 2 0 1 4 , ~ 8 : 0 0 - 9 : 0 0}\end{array}$ \\
\hline A70 & (no data) & (no data) & (no data) \\
\hline A7 & 1,46 & 4,02 & 2,54 \\
\hline A6 & 3,05 & 2,98 & 1,30 \\
\hline A73 & 0,78 & 1,98 & 2,03 \\
\hline A9 & (no data) & (no data) & (no data) \\
\hline A3 & (no data) & (no data) & (no data) \\
\hline A73b & (no data) & (no data) & (no data) \\
\hline A3b & 2,58 & 2,67 & 1,97 \\
\hline
\end{tabular}

According to Table 3, all segments present a GEH value less than five $(\mathrm{GEH}<5)$, meaning that we can safely assume that the two days are typical.

\section{Conclusions}

This paper proposed a systematic manual calibration process for a highway network model. The calibration was achieved into two stages. The first stage referred to a reasonable adjustment of the input data (desired speeds) in order to minimize the divergence from the field data (BT travel times), while the second stage referred to the selection of the optimal parameter set. In order to conclude to the optimal parameter set, a certain zone of suitable parameters' values was determined based on Xin (2013) and Rompis et al. (2014). The optimum values were selected based on a repetitive process of trial and error in the delimited zone.

The calibration results showed that the Vissim model was calibrated adequately under the acceptance targets that have been set. After five simulation runs, performed with random seeds (number of random seeds: $11,21,31,41,51)$, only two sections between BT 68-86 and 91-90 were exceeding the acceptance thresholds. However, in the section between BT 68-86, trucks are within the desired thresholds and cars exceeded only $0,7 \%$ and $0,5 \%$ the $15 \%$ limit during 8:00 am - 8:30 am and 8:30 - 9:00 am respectively, see Table 2. More specifically for this section, in three out of five simulation runs during 8:00 am - 8:30 am, the percentage of cars' deviation was below the $15 \%$ limit. Regarding the 8:30 am 9:00 am interval, maximum accepted deviation was not exceeded also in two simulation runs. Therefore, can be considered overall, that this section during 8:00 am - 9:00 am can also export reliable results. The level of detail, the scope of the actual simulation study and the overall needs of the simulation can determine whether this section in the upper interval should be included or not for further purposes.

A big disadvantage of systematic calibration procedures is the absence of an easy way to check the robustness of the calibration framework that is applied. The wide spectrum of simulation model cases should be classified based on models' similar elements, and then accordingly be calibrated by the use of the appropriate algorithm. Also, the development of traffic micro simulation parameter optimization tools, combining appropriate algorithms to find the optimum solution for every model, could be proved a very useful tool for models' calibration.

Concerning further research on this model, additional datasets on compatible 'typical' days would validate the simulation results and document the level of credibility for the segments of the network where BT detectors are still missing or an incident occurred. A potential adjustment of the heavy vehicle percentages would also raise the credibility and establish the compatibility of the datasets.

\section{References}

1. Chu, L., Liu, X. H., Oh, J-S. and Recker, W. (2004) A Calibration Procedure for Microscopic Traffic Simulation. 2004 TRB Annual Meeting, November 18.-20., 2004.

2. Dowling, R., Skabardonis, A., Halkias, J., McHale, G. and Zammit, G. (2004) Guidelines for Calibration of Microsimulation Models: Framework and Applications, Transportation Research Record: Journal of the Transportation Research Board, Vol. 1876, TRB, National Research Council, Washington D.C., 2004, pp. 1-9. 
3. Federal Highway Research Institute. (2011) Manual Traffic Census, available at: http://www.bast.de/DE/Statistik/Verkehrsdaten-Downloads/2010/zaehlung-2010-

BAB.pdf? blob=publicationFile \&v=1.

4. Forschungsgesellschaft fuer Strassen- und Verkehrswesen Arbeitsgruppe Verkehrsfuehrung und Verkehrssicherheit. (2006) Hinweise zur mikroskopischen Verkehrsflusssimulation.

5. Gomes, G., May, A. and Horowitz, R. (2004) Calibration of VISSIM for a Congested Freeway. California PATH Research Report UCB-ITS-PRR-2004-4. ISSN 1055-1425, March 2004.

6. Hourdakis, J., Michalopoulos, G. P. and Kottommannil, J. (2003) Practical Procedure for Calibrating Microscopic Traffic Simulation Models. 2003 TRB Annual Meeting, January 12-16., 2003.

7. Margreiter, M., Spangler, M., Zeh, T. and Carstensen, C. (2015) Bluetooth-Measured Travel Times for Dynamic Re-Routing. In: Proceedings of the 3rd Annual International Conference ACE 2015, Volume 2, Global Science and Technology Forum, Singapore, pp. 447.

8. Park, B. and Won, J. (2006) Microscopic Simulation Model Calibration and Validation Handbook, University of Virginia, Charlottesville, Virginia Transportation Research Council, Charlottesville, available at: http://www.virginiadot.org/vtrc/main/online_reports/pdf/07-cr6.pdf

9. Paz, A., Molano, V., Martinez, E., Gaviria, C., Arteaga, C. (2015) Calibration of Traffic flow models using a memetic algorithm, Transportation Research Part C, Vol. 55, pp. 432-443.

10. PTV Group. (2014) Vissim 7 User Manual.

11. Rompis, S., Habtemichael, F., and Cetin, M. (2014) A Methodology for Calibrating Microscopic Simulation for Modeling Traffic Flow under Incidents, 2014 IEEE 17th International Conference on Intelligent Transportation Systems (ITSC) October 8-11, 2014. Qingdao, China.

12. So, J., Ostojic, M., Stevanovic, A., Sajjadi, S., Jolovic, D. (2015) Building, Calibrating and Validating a Large-Scale High-Fidelity Microscopic Traffic simulation Model - A Manual Approach, 95th Transportation Research Board Annual Meeting Proceedings, 2015.

13. Wisconsin Department of Transportation. (2002) Microsimulion Guidelines, available at: http://www.wisdot.info/microsimulation/index.php?title=Model_Calibration\#The_GEH_Formula

14. Xin, F. (2013) Entwicklung eines Kalibrierungsverfahrens für $\bar{M}$ ikrosimulationen auf $\bar{d}$ er Basis von Reisezeitdaten mit Demonstration auf Autobahnen im Norden Muenchens. Master's Thesis, Chair of Traffic Engineering and Control, Technische Universitaet Muenchen.

15. Young, E. S. (2012) Bluetooth traffic detectors for use as permanently installed travel time instruments, available at: file:///F:/Master\%20Thesis\%20new/Kaio/Papers\%20for\%20callibration/BLUETOOTHTRAFFIC-DETECTORS-FOR-USE-AS-PERMANENTLY_X.pdf 